Kansas State University Libraries

New Prairie Press

\title{
CONSTRUCTION OF ASYMMETRICAL RESPONSE SURFACE DESIGNS
}

V. P. Manocha

L. S. Kaushik

Follow this and additional works at: https://newprairiepress.org/agstatconference

Part of the Agriculture Commons, and the Applied Statistics Commons

\section{c) (i) $९$}

This work is licensed under a Creative Commons Attribution-Noncommercial-No Derivative Works 4.0 License.

\section{Recommended Citation}

Manocha, V. P . and Kaushik, L. S. (1998). "CONSTRUCTION OF ASYMMETRICAL RESPONSE SURFACE DESIGNS," Conference on Applied Statistics in Agriculture. https://doi.org/10.4148/2475-7772.1285

This is brought to you for free and open access by the Conferences at New Prairie Press. It has been accepted for inclusion in Conference on Applied Statistics in Agriculture by an authorized administrator of New Prairie Press. For more information, please contact cads@k-state.edu. 


\title{
Construction of Asymmetrical Response Surface Designs
}

\author{
V. P.Manocha and L. S. Kaushik \\ Department of Maths \& Stats., \\ CCS Haryana Agricultural University, Hisar-125004
}

\begin{abstract}
The paper proposes several methods for constructing both rotatable and non-rotatable asymmetrical response surface designs. The idea of modified rotatable design is introduced. In most of the experiments conducted by the experimenter it is not necessary that all the factors under study may have equal number of levels The methods proposed will have wider use under these circumstances.
\end{abstract}

Key words: Response surface design, symmetrical response surface design, asymmetrical response surface design, , factorial, fractional factorial, rotatable, non-rotatable, second order rotatable design, modified rotatable .

\section{Introduction}

Response surface designs are essentially factorial designs. These are used to fit suitable surfaces. Here it is assumed that the response from a treatment combination is a function of the main effects and interaction of the different factors. The importance of this type of study is that we can know the variation in response with the corresponding change in the level of application of a group of factors. The functional relationship may be of any degree. But we shall consider second degree polynomial. The equation of polynomial surface relation when there are $\mathrm{v}$ factors and $\mathrm{N}$ observations in a design is written as :-

$$
y_{u}=b_{0}+\sum_{i}^{v} b_{i} x_{i u}+\sum_{i}^{v} b_{i l} x_{i u}^{2}+\sum_{i}^{v} \sum_{j>i}^{v} b_{i j} x_{i u} x_{j u}+e_{u}
$$

Where $\mathrm{y}_{\mathrm{u}}$ stands for response of the $\mathrm{u}$-th observation and $\left(X_{i u}-\bar{X}_{i}\right) / c_{i}$, $(\mathrm{i}=1,2, \ldots \mathrm{N})$. Further $\mathrm{X}_{\mathrm{iu}}$ is the original level of the $\mathrm{i}$-th factor in the u-th treatment and $\bar{X}_{i}$ is the mean of the levels of the $i$-th factor. Here $X_{\mathrm{iu}}$ 's are the dose codes and it will be seen that the surface relation in (1.1) has been written in terms of dose codes. $\mathrm{e}_{\mathrm{u}}$ 's are assumed as independent normal variables with mean 0 and constant variance $\sigma^{2}$.

$$
\text { Let } \quad\left(y_{u}\right)_{E}=b_{0}+\sum_{i}^{v} b_{i} x_{i u}+\sum_{i}^{v} b_{i i} x_{i u}^{2}+\sum_{i}^{v} \sum_{j>i}^{v} b_{i j} x_{i i u} x_{j u}
$$




$$
\text { Then, } \quad y_{u}=\left(y_{u}\right)_{E}+e_{u}
$$

Here $y_{u}$ is the observed response and $\left(y_{u}\right)_{E}$ can be considered the response expected from the relation corresponding to $\mathrm{y}_{\mathrm{u}}$.

$$
\text { Then, } \quad E=\left[y_{u}+\left(y_{u}\right)_{E}\right]^{2}
$$

and the ${ }^{\mathrm{v}+2} \mathrm{C}_{2}$ normal equations ( ${ }^{\mathrm{v}} \mathrm{C}_{0}$ for $\mathrm{b}_{0},{ }^{\mathrm{v}} \mathrm{C}_{1}$ for $\mathrm{b}_{\mathrm{i}}$ 's and $\mathrm{b}_{\mathrm{ii}}$ 's each and ${ }^{\mathrm{v}} \mathrm{C}_{2}$ for $\mathrm{b}_{\mathrm{ij}}$ 's) are obtained by equating the partial derivatives of $E$ with respect to the parameters $b_{0}, b_{i}, b_{i i}, b_{i j}$ to zero. It will be seen that the normal equations contain beside the parameters, the sum of products like

$$
P_{p q r}=\sum x_{i u}^{p} x_{j u}^{q} x_{k u}^{r} \ldots
$$

where

(1) $\mathrm{p}, \mathrm{q}, \mathrm{r}$ etc., can each take values from 0 to 4 .

(2) $\operatorname{sum} \mathrm{p}+\mathrm{q}+\mathrm{r}+\ldots \leq 4$.

(3) i, j, k etc., need not all be unequal.

A response surface design consists of a number of $N$ treatments (observations) such that

$$
P_{p q r}=0
$$

when at least one of $\mathrm{p}, \mathrm{q}, \mathrm{r} \ldots$ is odd and $\mathrm{i}, \mathrm{j}, \mathrm{k}$ 's are unequal. This property simplifies solution of the normal equations greatly. A further simplification is obtained when

$$
P_{p q r}=\text { constant }
$$

each $\mathrm{p}, \mathrm{q}, \mathrm{r} \ldots$ is even not all zero. Here the value of the constant depends upon the sum $\mathrm{p}+\mathrm{q}+\mathrm{r}+$. $\quad$ und e r t h e se considerations $\sum x_{i u}=0, \sum x_{i u} x_{j u}=0, \sum x_{i u}^{3}=0, \sum x_{i u}^{2} x_{j u}=0, \sum x_{i u} x_{j u} x_{k u} x_{l u}=0$ and these are some of the sums which appear in the normal equations.

Further, let

$$
\begin{gathered}
\sum x_{i u}^{2}=\text { constant say, } R \\
\sum x_{i u}^{2} x_{j u}^{2}=\text { constant say, } L \\
\sum x_{i u}^{4}=\text { constant, say, } C L \text { where } C \text { is a constant } .
\end{gathered}
$$

Though considerable work exists in literature regarding symmetrical response surface designs particularly the rotatable series, no significant work seems to have been done on asymmetrical response surface designs. This paper proposes some methods for the construction of both rotatable and non-rotatable asymmetrical response surface designs.

Our approach is first to select the dose-code of the factors such that the condition $\mathrm{P}_{\mathrm{pqr}}=$ 
0 for at least one of $\mathrm{p}, \mathrm{q}, \mathrm{r}$ as odd is satisfied. This is done as follows.

Given the range of the levels of a factor, a level at or near the middle of the range is fixed. If this level is denoted by $M$, then levels like $M \pm k$ are chosen for the factor. $k$ is chosen so that $\mathrm{M}-\mathrm{k}$ is greater than or equal to the minimum level in the level range and $\mathrm{M}+\mathrm{k}$ is less that or equal to the maximum level defining the range. Such choice of k gives two levels M-k and $\mathrm{M}+\mathrm{k}$ and there can be as many choices of $\mathrm{k}$ as required. If $\mathrm{k}$ is zero, then $\mathrm{M}$ is also a level and this does not pair with any other level. The number of levels in this case becomes odd and when $\mathrm{M}$ is not a level the number of levels is even. These levels are transformed to dose codes by first shifting the origin to $\mathrm{M}$ and then dividing the deviates by a suitable scaling constant. The case of equispaced levels is a particular case of this more general procedure. But the requirements (i) $\sum \mathrm{x}_{\mathrm{iu}}{ }^{2}=$ constant (ii) $\sum \mathrm{x}_{\mathrm{iu}}{ }^{2} \mathrm{x}_{\mathrm{ju}}{ }^{2}=$ constant (iii) $\sum \mathrm{x}_{\mathrm{iu}}{ }^{4}=$ constant are not automatically satisfied. We shall devise methods to satisfy the condition by putting further restrictions on level selection.

\section{Review of Literature}

\subsection{Symmetrical Response Surface Designs}

So far as the contribution of design of experiments in the investigation of any response surface relationship is concerned, Yates(1935) was the first to introduce the use of factorial designs with two or more factors, each including at least three levels. Plackett and Burman(1946) gave a generalized idea regarding the use of fractional factorial designs although the original idea was from Finney(1945). Box and Wilson (1951) were the first to give some useful first and second order designs.

Box and Hunter(1957) introduced the concept of rotatable designs for fitting second degree response surfaces. Box Behnken (1960b)got Second Order Rotatable Design(SORD) using BIBD, [An incomplete block design with $v$ treatments distributed over $b$ block, each of size $\mathrm{k}$, where $\mathrm{k}$ is less than $\mathrm{v}$ such that each treatment occurs in $\mathrm{r}$ blocks, no treatment occurs more than once in a block and each pair of treatments occurs together in $\lambda$ blocks, is called a balanced incomplete block designs BIBD], with $r=3 \lambda$, for $v$ factors each at 3 levels. Raghavarao(1963) got the same for $r<3$. Herzberg(1966) generalized the concept of rotatability and introduced a series of response surface designs, which were called Cylindrically Rotatable Designs. These designs are rotatable with respect to all factors except one.

\subsection{Asymmetrical Response Surface Designs}

Ramchander (1963), first attempted to obtain some asymmetrical response surface designs. He obtained two series of such designs which were incomplete factorials of the type $3 \times 5^{\mathrm{m}}$ and can be used for fitting second degree response surfaces. However, these designs do not satisfy the criterion of constructing asymmetrical rotatable designs. Dey(1969) discussed 
methods of construction of second order asymmetric response designs, both rotatable and nonrotatable, and constructed the designs of the form $3^{\mathrm{n}} \times 5^{\mathrm{m}}$. It has been shown by him that these designs ensures rotatability for those factors which are at 5 levels, though the design as a whole is non-rotatable. He called these designs Partially Rotatable Designs.

\section{Designs of the form $3 x^{2}$}

Here, there are three factors, say, A, B and C with number of levels $3, \mathrm{~s}, \mathrm{~s}$ respectively, where $\mathrm{s}>3$. The dose codes for $\mathrm{A}$ are taken as $-\mathrm{a}, 0, \mathrm{a}$ and for each of $\mathrm{B}$ and $\mathrm{C}$ are taken as $\left(-\mathrm{kb},-\mathrm{d}_{1} \mathrm{k},-\mathrm{d}_{2} \mathrm{k}, \ldots \mathrm{d}_{2} \mathrm{k}, \mathrm{d}_{1} \mathrm{k}, \mathrm{kb}\right)$ where $\mathrm{k}$ is an unknown dose code and $\mathrm{d}_{1}, \mathrm{~d}_{2}, \ldots$ .are known dose codes such that $\sum \mathrm{d}_{\mathrm{ij}}{ }^{2}=\mathrm{s}_{2 \mathrm{i}}$ and $\sum \mathrm{d}_{\mathrm{ij}}{ }^{4}=\mathrm{s}_{4 \mathrm{i}}$, where $\mathrm{i}$ stands for the factor and the summation $\mathrm{j}$, where $\mathrm{j}$ varies from 1 to the number of positive doses. If $\mathrm{s}$ is odd, a zero dose code is there. Here $\mathrm{a}$ and $\mathrm{b}$ are unknown scaling constants of doses.

Let $\mathrm{N}=3 \mathrm{xs}^{2}$. The design consists of $\mathrm{N}$ points obtainable from the above dose codes of three factors.

$$
\sum x_{i u}^{2}=\frac{2 N}{3} a^{2}
$$

This result follows from the fact that in the design the dose a of the first factor occurs $\mathrm{s}^{2}$ times and so also the dose -a. Hence the sum of squares of the doses of the first factor in the $\mathrm{N}$ treatments in the design is

$$
\begin{gathered}
2 a^{2} s^{2}=\frac{N x 2 a^{2}}{3} \text { as } s^{2}=\frac{N}{3} \text { from the design. } \\
\sum x_{2 u}=\frac{2 N\left(s_{22}+K^{2}\right) b^{2}}{s} \\
\sum x_{3 u}=\frac{2 N\left(s_{23}+K^{2}\right) b^{2}}{s}
\end{gathered}
$$

as $s_{22}$ and $s_{23}$ are equal as each corresponding factor has $s$ levels. We shall take $s_{22}=s_{23}=s_{2}$ In general for any two factors $\mathrm{i}$ and $\mathrm{j}$

$$
\sum x_{i u}^{2} x_{j u}^{2}=\frac{4 N\left(s_{2 i}+K_{i}^{2}\right)\left(s_{2 j}+K_{j}^{2}\right) a_{i}^{2} a_{j}^{2}}{s_{i} s_{j}}
$$

where $s_{i}$ and $s_{j}$ are the numbers of levels of the $i$-th and $j$-th factors respectively, $s_{2 i}+k_{i}^{2}$ is the sum of squares of the levels codes of the $i$-th factor. Similarly $s_{2 j}+k_{j}^{2}$ is the sum of squares of the levels codes of the $\mathrm{j}$-th factor, $\mathrm{a}_{\mathrm{i}}$ is the scaling factor of the doses of the $\mathrm{i}$-th factor and $\mathrm{a}_{\mathrm{j}}$ is 
the scaling factor of the doses of the $\mathrm{j}$-th factor.

In the present design $s_{21}=1$ and $k_{1}=0$ as its levels are $-1,0,1$ with scaling constant a.

Hence

$$
\begin{aligned}
& \sum x_{1 u}^{2} x_{2 u}^{2}=\frac{2 N \times 2\left(s_{2}+K^{2}\right) a^{2} b^{2}}{3 s} \\
& \sum x_{1 u}^{2} x_{3 u}^{2}=\frac{2 N x 2\left(s_{2}+k^{2}\right) a^{2} b^{2}}{3 s} \\
& \sum x_{2 u}^{2} x_{3 u}^{2}=\frac{2 N x 4\left(s_{2}+k^{2}\right) b^{4}}{s^{2}}
\end{aligned}
$$

The scaling constant has been taken as b for each of the last two factors as each of them has same doses.

$$
\begin{aligned}
& \text { Also, } \quad \sum x_{1 u}^{4}=\frac{N x 2 a^{4}}{3} \\
& \sum x_{2 u}^{4}=\frac{2 N\left(s_{4}+k^{4}\right) b^{4}}{s} \\
& \sum x_{3 u}^{4}=\frac{2 N\left(s_{4}+k^{4}\right) b^{4}}{s}
\end{aligned}
$$

Here $s_{4}+k^{4}$ is the sum to the power four of the doses of each of the factors at s levels.

Now,

$$
\begin{aligned}
\sum x_{1 u}^{2} & =\sum x_{2 u}^{2} \\
\text { if } \quad \frac{2 N}{3} a^{2} & =\frac{2 N\left(s_{2}+k K^{2}\right) b^{2}}{s} \\
\text { Then, } \quad \frac{2}{3} a^{2} & =\frac{2\left(s_{2}+k^{2}\right) b^{2}}{s}
\end{aligned}
$$

This is also the condition for $\sum \mathrm{x}_{1 \mathrm{u}}{ }^{2}=\sum \mathrm{x}_{3 \mathrm{u}}{ }^{2}$

Again

$$
\sum x_{1 u}^{2} x_{2 u}^{2}=\sum x_{2 u}^{2} x_{3 u}^{2}
$$




$$
\begin{aligned}
& \text { if } \frac{2 N \times 2\left(s_{2}+k^{2}\right) a^{2} b^{2}}{3 s}=\frac{N x 4\left(s_{2}+k^{2}\right)^{2} b^{4}}{s^{2}} \\
& \text { i.e. } \quad \frac{2}{3} a^{2}=\frac{2\left(s_{2}+k^{2}\right) b^{2}}{s}
\end{aligned}
$$

But it will be seen from (3.10) that this is also the condition to make $\sum x_{1 u}^{2}=\sum x_{2 u}^{2}$. This shows that if the condition $\sum x_{1 u}^{2}=\sum x_{2 u}^{2}$ holds then the condition $\sum x_{1 u}^{2} x_{2 u}^{2}=\sum x_{2 u}^{2} x_{3 u}^{2}$.also holds.

Hence $\sum x_{i u}^{2}$ is made constant by suitable choice of the unknown level $\mathrm{k}_{\mathrm{i}}$, the constancy of $\sum x_{i u}^{2} x_{j u}^{2}$ is automatically taken care of. This means if $\mathrm{R}$ is constant for the factors then $\mathrm{L}$ is also constant over different pairs of factors. Further it can be shown below that $L=R^{2} / N$ for complete factorial.

In the design under consideration

$$
\begin{gathered}
\qquad R=\sum x_{i u}^{2}=\frac{2 N}{3} a^{2} \\
=\text { constant over the factors subject to (3.10). } \\
L=\frac{4 N\left(s_{2}+k^{2}\right) a^{2} b^{2}}{3 s} \\
=\frac{4 N}{9} a^{4} \text { eliminating }\left(s_{2}+k^{2}\right) b^{2} \text { using (3.10). }
\end{gathered}
$$

Now,

$$
\sum x_{1 u}^{4}=\sum x_{2 u}^{4}
$$

From (3.10) we require

$$
\begin{gathered}
\frac{a^{2}}{b^{2}}=\frac{2 \times 3\left(s_{2}+k^{2}\right)}{s \times 2} \\
\text { if } \quad \frac{2 N}{s} a^{4}=\frac{2 N\left(s_{4}+k^{4}\right) b^{4}}{s^{2}}
\end{gathered}
$$




$$
\text { i.e., if } \begin{aligned}
\frac{a^{4}}{b^{4}} & =\frac{2 \times 3\left(s_{4}+k^{4}\right)}{s \times 2} \\
& =\frac{3\left(s_{4}+k^{4}\right)}{s}
\end{aligned}
$$

Eliminating a and b using (3.11) and (3.12), we get;

$$
\begin{gathered}
\frac{3 \times 2\left(s_{4}+k^{4}\right)}{s \times 2}=\frac{4 \times 9\left(s_{2}+k^{2}\right)^{2}}{4 s^{2}} \\
\text { i.e. } \quad s\left(s_{4}+k^{4}\right)=3\left(s_{2}+k^{2}\right)^{2} \\
\text { i.e., } \quad k^{4}(s-3)-6 s_{2} k^{2}+s x s_{4}-3 s_{2}^{2}=0
\end{gathered}
$$

This is a quadratic equation in $k^{2}$, the square of the unknown dose code. By solving the equation we get $\mathrm{k}^{2}$. Once $\mathrm{k}$ is known the design is known. In this design all the conditions except $\mathrm{C}=3$ are satisfied as in the symmetrical response surface design. In these designs $\mathrm{R}^{2}=\mathrm{NL}$ also holds as shown above. Before we discuss further we take a particular case viz., $\mathrm{s}=4$.

\subsection{Designs of the form $3 \times 4^{2}$}

Here the doses are $-\mathrm{a}, 0$, a for $\mathrm{A},-\mathrm{kb},-\mathrm{b}, \mathrm{b}, \mathrm{kb}$ for each of $\mathrm{B}$ and $\mathrm{C}, \mathrm{k}$ is unknown.

Here $s_{21}=1, s_{22}=s_{23}=s_{2}=\left(k^{2}+1\right) ; s_{41}=1$ and $s_{42}=s_{24}=s_{4}=\left(k^{4}+1\right)$;

$$
R=\frac{2 N}{3} a^{2}
$$

Further from (3.11)

$$
\begin{aligned}
L=\frac{4 N}{8} a^{4} \text { subject to } \frac{a^{2}}{b^{2}} & =\frac{3 \times 2\left(1+k^{2}\right)}{8} \text { as in } \\
C L & =\frac{2 N}{3} a^{4} \\
\frac{a^{4}}{b^{4}} & =\frac{3\left(1+k^{4}\right)}{4}
\end{aligned}
$$

Eliminating $\mathrm{a}^{2} / \mathrm{b}^{2}$ from (3.14) and (3.15), we get; 


\section{Applied Statistics in Agriculture}

$$
\begin{aligned}
& \qquad \frac{9\left(1+k^{2}\right)^{2}}{16}=\frac{3\left(1+k^{4}\right)}{4} \\
& \text { i.e. } \quad 3\left(1+k^{2}\right)^{2}=4\left(1+k^{4}\right) \\
& \text { i.e. } \quad k^{4}-6 k^{2}+1=0
\end{aligned}
$$

This equation also follows from (3.13) taking $s_{2}=2, s_{4}=2, s=4$; after solving we, get

$$
k^{2}=\frac{6 \pm \sqrt{32}}{2}=3 \pm 2 \sqrt{2}
$$

Both the solutions are positive. Thus, we get two designs one for each value of $\mathrm{k}$. We know $k=a / b$. We can fix one of $a$ and $b$ arbitrarily and taking $a=1$, we get $b=1 / k$.

This design can be said to be Modified Asymmetrical Response Surface Design because the design satisfies $\mathrm{R}^{2}=\mathrm{NL}$

\subsection{Rotatable Asymmetrical Response Surface Design}

We take the same design as above with their new known dose-codes as obtained from the solution of the equation. In this design $\mathrm{P}_{\mathrm{pqr}}=0$ for odd $\mathrm{p}$ and $\mathrm{P}_{\mathrm{pqr}}=$ constant for even $\mathrm{p}$ ' $\mathrm{s}$. We include $2 \mathrm{v}$ points more in the design obtainable from the sets $(\mathrm{d}, 0,0),(0, \mathrm{~d}, 0),(0,0, \mathrm{~d})$. We have

$$
\begin{gathered}
R_{1}=\frac{2 N}{3} a^{2}+2 d^{2} \\
L_{1}=\frac{4 N}{9} a^{4} \\
N_{1}=\text { No. of observations }=N+6
\end{gathered}
$$

To make the design rotatable we require

$$
\begin{aligned}
& \frac{2 N}{3} a^{4}+2 d^{4}=3 \frac{4 N}{9} a^{4} \\
& \text { i.e. } \quad d^{4}=\frac{N}{9} a^{4}=s^{2} a^{4} \\
& \text { and } \quad d^{2}=s a^{2}
\end{aligned}
$$

Hence, the unknown level of $d$ is known and for this value of $d$ the design is Rotatable. 


\subsection{Modified Rotatable Design}

Now we define a design to be Modified Rotatable Design if it satisfies the condition $\mathrm{R}^{2}=\mathrm{NL}$

The design given in (3.2) does not satisfy the condition $R_{1}^{2}=N_{1} L$.

$$
R_{1}=\frac{2 N}{3} a^{2}+d^{2} \quad \text { and } \quad N_{1}=N+6
$$

This condition is satisfied by including a requisite number of central points. In the rotatable design, we get $d^{2}=s^{2}$ as in (3.20). Substituting this value of $d^{2}$ in (3.17), we get

$$
R_{1}=\frac{2 N}{3}+2 s a^{2}=2 s a^{2}(s+1)
$$

For the condition $\mathrm{R}_{1}{ }^{2}=\mathrm{N}_{2} \mathrm{~L}$ to hold, we require

$$
N_{2}=\frac{R_{1}^{2}}{L}=\frac{4 s^{2} a^{4}(s+1)^{2} \times 9}{4 N a^{4}}=3(s+1)^{2}
$$

In the rotatable design, we have $\mathrm{N}_{1}=\mathrm{n}+6, \mathrm{~N}=3 \mathrm{~s}^{2}$. Therefore, the number of central points to be included is $\mathrm{N}_{2}-\mathrm{N}_{1}=3(2 \mathrm{~s}-1)$. Thus, we get a series of designs which satisfy both the conditions viz., $\mathrm{C}=3$ and $\mathrm{R}_{1}{ }^{2}=\mathrm{N}_{2} \mathrm{~L}$ obtainable by including $3(2 \mathrm{~s}-1)$ central points.

\section{Example.}

We continue with the design taken in (3.2). We have in (3.19)

$$
d=\sqrt{4 a^{2}}=2 a
$$

Hence the extra sets $(d, 0,0),(0, d, 0)$ and $(0,0, d)$ become $(2,0,0),(0,2,0)$ and $(0,0,2)$ when $\mathrm{a}=1$. The number of central points to be included are $3(2 \mathrm{~s}-1)=21$ and the number of points in the design are $\mathrm{N}_{2}=75, \mathrm{~N}=48, \mathrm{~N}_{1}=\mathrm{N}+6=54$.

\subsection{Modified Design for any number of factors with first factor at 3 levels}

We have seen in (3.19) that

$$
\text { We have seen in (3.19) that } d^{4}=\frac{N}{3} a^{4}
$$

We need $\mathrm{R}$ in (3.17) as an integer after substituting the value of $\mathrm{d}$ as obtained in (3.20). This is possible when $N / 3$ is a perfect square. Keeping this in view was taken a $3 \mathrm{~s}^{2}$ as a matter of fact all the results will hold when $N / 3$ is a perfect square where 


$$
\frac{N}{3}=s^{2 m} x s_{1}^{2 m_{1}}
$$

Thus, this technique holds for any number of factors in groups such that each group of factors contains an even number of factors each having the same number of levels. Even the number of factors each with 3 factors may be more than one but an odd number. Further, factors with 4 levels can be taken any number of times and not necessarily an even number of times as 4 itself is a perfect square.

We consider the design $3^{3} \times 4^{2} \times 5^{2}$ with levels $-a, 0$, a for each of the first 3 factors, $-k_{1} b_{1},-b_{1}$, $b_{1}, k_{1} b_{1}$ and $-k_{2} b_{2}-b_{2}, b_{2}, k_{2} b_{2}$ for each of the factors with 5 levels.

Here

$$
R=\frac{2 N}{3} a^{2}, \quad L=\frac{4 N}{9} a^{4}, \quad C L=\frac{2 N}{3} a^{4}
$$

subject to

$$
\begin{gathered}
\frac{2 N}{3} a^{2}=\frac{2 N\left(1+k_{1}^{2}\right) b_{1}^{2}}{4}=\frac{2 N\left(1+k_{2}^{2}\right) b_{2}^{2}}{5} \\
\text { i.e., } \quad \frac{a^{2}}{b_{1}^{2}}=\frac{3\left(1+k_{1}^{2}\right)}{4} \\
\frac{a^{2}}{b_{2}^{2}}=\frac{3\left(1+k_{2}^{2}\right)}{5}
\end{gathered}
$$

also subject to

$$
\begin{aligned}
\frac{2 N}{3} a^{4}=\frac{\left.2 N\left(1+k_{1}^{4}\right) b_{1}^{4}\right)}{4} & =\frac{2 N\left(1+k_{2}^{4}\right) b_{2}^{4}}{5} \\
\text { i.e., } \quad \frac{a^{4}}{b_{1}^{4}} & =\frac{3\left(1+k_{1}^{4}\right)}{4} \\
\text { i.e., } \quad \frac{a^{2}}{b_{2}^{4}} & =\frac{3\left(1+k_{2}^{4}\right)}{5}
\end{aligned}
$$

Eliminating $\mathrm{a}^{2} / \mathrm{b}_{1}^{2}$ using (3.21) and (3.23) we get a quadratic equation in $\mathrm{k}_{1}^{2}$ and its solution gives $\mathrm{k}_{1}$. Similarly eliminating $\mathrm{a}^{2} / \mathrm{b}_{2}^{2}$ using (3.22) and (3.24) we get another quadratic equation 
in $\mathrm{k}_{2}^{2}$ and its solution gives with $\mathrm{k}_{2}$ with these values of $\mathrm{k}_{1}$ and $\mathrm{k}_{2}$ the condition $\mathrm{R}^{2}=\mathrm{NL}$ holds and as such these designs are Modified Response Surface designs.

\subsection{Modified Rotatable Response Surface design from the designs in previous section}

We take further $\mathrm{v}$ sets $(\mathrm{d}, 0,0, \ldots 0)$, where $\mathrm{v}$ is the number of factors in the design.

With these sets

$$
\begin{gathered}
R_{1}=R+2 d^{2} \\
L_{1}=L=\frac{4 N}{9} a^{4}=\frac{4 \times 3^{3} \times 4^{2} \times 5^{2}}{9} a^{4}=12 \times 4^{2} \times 5^{2} a^{4} \\
C l_{1}=C l L+2 d^{4}
\end{gathered}
$$

To make the design rotatable, we need

$$
\begin{aligned}
& \quad C l L+2 d^{4}=3 L \\
& \text { i.e., } \quad \frac{2 N}{3} a^{4}+2 d^{4}=\frac{3 x 4 N}{9} a^{4} \\
& \text { i.e. } \quad d^{4}=\frac{N}{3} a^{4}=3^{2} \times 4^{2} \times 5^{2} a^{4} \\
& \text { and } \quad d^{2}=3 \times 4 \times 5 \times a^{2}
\end{aligned}
$$

Hence

$$
R_{1}=\frac{2 N}{3} a^{2}+2 \times 3 \times 4 \times 5 \times a^{2}=\left(2 \times 3^{2} \times 4^{2} \times 5^{2}+2 \times 3 \times 4 \times 5\right) a^{2}=2 \times 3 \times 4 \times 5(3 \times 4 \times 5+1) a^{2}
$$

Now for $R_{1}^{2}=N_{2} L_{1}$ to hold we require

$$
N_{2}=\frac{R_{1}^{2}}{L}=\frac{(120)^{2} \times(61)^{2} \times a^{4}}{12 \times 16 \times 25 x a^{4}}=61 \times 61 \times 3
$$

In this case $363-14=349$ central points are needed to make the design modified rotatable with $\mathrm{N}=10800$. Such designs can have, at present only illustrative use to substantiate the method in all its facets. A more reasonable design is $3 \times 4 \times 5^{2}$.

For this design 


$$
\begin{gathered}
d^{4}=4 \times 5^{2} x a^{4} \\
d^{2}=10 a^{2} \\
R_{1}=220 a^{2} \\
L_{1}=\frac{4^{2} \times 5^{2} \times a^{4}}{3} \\
N_{2}=\frac{220 \times 220 \times 3}{4^{2} \times 5^{2}} \\
=363 \\
N_{1}=308
\end{gathered}
$$

This technique of getting Modified Rotatable Designs including central points could not be extended to other types of factor levels since the condition has to be the perfect square and this condition could not be satisfied. Here, $n$ is the number of levels of the factor with least number of levels greater than 3 . However, the modified design and rotatable design are available for any design and the methods for obtaining them were discussed previously.

\section{Conversion of dose codes to levels}

It will be seen that the design has been written using dose codes. But while adopting a design it is necessary to write them in terms of original levels that the users understand. The dose codes can be converted to the levels by using a linear relation connecting the dose codes and the levels. Let $\mathrm{B}$ and $-\mathrm{B}$ denote the highest and lowest dose codes in a design corresponding to the extreme levels defining the range of levels of a factor. Let the two extreme levels be denoted by $M_{x}$ and $M_{L}$. Then the line of dose code with levels passes through the points $\left(B, M_{x}\right)$ and $\left(-B, M_{L}\right)$. The equation of the line is

$$
\hat{y}=a_{1}+b_{1} x \text {, where } a_{1}=\frac{M_{x}+M_{L}}{2} \text { and } b_{1}=\frac{M_{x}-M_{L}}{2 B}
$$

Here $y$ stands for the level and $\mathrm{x}$ for the dose code. Substituting any dose-code, $\mathrm{x}$ in the above relation, the corresponding level can be obtained.

\section{Summary}

In most of the experiments conducted by the experimenter all the factors under study are not necessarily of equal number of levels due to various constraint. Having this in mind a general procedure for constructing rotatable and non-rotatable asymmetrical designs is given by imposing restrictions on the levels of the factors. By using this procedure series of rotatable and non-rotatable asymmetrical response surface designs by taking factors at different number of levels have been constructed. The concept of modified rotatable has been introduced. 


\section{References}

Box, G.E.P. and Benhken, D.W. (1960b). Some new three level designs for study of quantitative variables Technometrics. 2: 455-475.

Box, G.E.P. and Hunter, J.S. (1957). Multifactor experimental designs for exploring response surfaces. Ann. Math. Stat. 28: 195-241.

Box G.E.P. and Wilson, K.B. (1951). On the experimental attainment of optimum conditions ( with discussion). Jour. Roy. Stat. Soc. series B. 13: 1-45.

Dey, A. (1969). Some investigations on response surface designs. An unpublished Ph.D. thesis, Indian Agricultural Research Institute, New Delhi.

Finney, D.J. (1945). The fractional replications of factorial arrangements. Ann. Eugen. 12: 291-301.

Herzberg, A.M. (1966). Cylindrically rotatable designs of types 1, 2 and 3. Ann. Math. Stat. 38: 167-176.

Mehta, J.S. and Das, M.N. (1968). Asymmetric rotatable designs and orthogonal transformations. Technometrics 10: 313-322.

Plackett, R.L. and Burman, J.P. (1946). The design of multifactorial experiments. Biometrika 33: $305-325$.

Raghavarao, D. (1963). Construction of second order rotatable designs through incomplete block designs. jour. Ind. Soc. Assn. 1: 221.

Ramchander, P.R. (1963). Asymmetrical response surface designs. An unpublished diploma thesis, IASRI, New Delhi.

Yates, F. (1935). Complex experiments. J. Roy. Stat. Soc. 2(suppl.) : 181-247. 